\title{
Mecanobiología de los huesos maxilares. I. Conceptos generales
}

\author{
Cano J*, Campo J**, Palacios B***, Bascones A****
}

\section{RESUMEN}

La mecanobiología ósea se encarga de la interacción entre las señales mecánicas y los mecanismos moleculares en las células y el tejido óseo. El estudio actual de esta disciplina engloba los modelos informáticos, la biología molecular y las técnicas de imagen en alta resolución. En este artículo se revisan los conceptos generales que se estudian en la mecanobiología y biomecánica de los hueso maxilares. Se establecen las principales propiedades biomecánicas del hueso en las diferentes escalas de medición y determinados factores que influyen en la reacción del hueso perimplantario ante las cargas biomecánicas.

Palabras clave: Mecanobiología, hueso, implantes.

\section{SUMMARY}

Bone mechanobiology deals with connection between mechanical signals and molecular events in cells and bone tissue. The current study on this subject involves computer models, molecular biology and high resolution imaging of bone. This paper reviews general concepts which take place on mechanobiology and biomechanics of maxillary bones. Mechanical features in several dimensional levels are studied and some factors with influence on perimplantary bone are also evaluated.

Key words: Mechanobiology, bone, implants.

Fecha de recepción: Febrero 2007.

Aceptado para publicación: Abril 2007.

* Profesor Asociado. Departamento de Medicina y Cirugía Bucofacial. Facultad de Odontología. UCM.

** Profesor Contratado Doctor. Departamento de Medicina y Cirugía Bucofacial. Facultad de Odontología. UCM.

*** Prof. Colaboradora. Departamento de Medicina y Cirugía Bucofacial. Facultad de Odontología. UCM.

**** Catedrático de Medicina y Cirugía Bucofacial. Departamento de Estomatología III. Facultad de Odontología. UCM.

Cano J, Campo J, Palacios B, Bascones A. Mecanobiología de los huesos maxilares. I. Conceptos generales. Av. Odontoestomatol 2007; 23 (6): 347-358.

\section{INTRODUCCIÓN}

La mecanobiología estudia la interacción entre las señales mecánicas y los procesos biológicos que se producen en las células y tejidos. La carga mecánica puede influir en la proliferación, diferenciación y metabolismo celular, por lo que tiene un papel cru- cial en el crecimiento, adaptación, regeneración y bioingeniería de los tejidos vivos. La mecanobiología combina técnicas biológicas experimentales (modelos in vitro e in vivo) y técnicas computarizadas (modelos matemáticos e informáticos) para crear la interacción entre la mecánica y la biología. Desde el punto de vista biomecánico, ningún tejido vivo pue- 
de compararse a otro material de ingeniería, ya que dichos tejidos presentan un continuo proceso de regeneración y remodelación que permite mejorar su estructura en función de los esfuerzos a los que es sometido (1).

Van der Meulen describió la mecanobiología esquelética como "la ciencia que estudia las fuerzas mecánicas que modulan la morfología y la adaptación estructural de los tejidos esqueléticos; es decir, hueso, cartílago, ligamento y tendón" (2). Existen tres aspectos que han hecho avanzar enormemente la mecanobiología ósea en los últimos años:

a) Los modelos informáticos de estructuras que permiten el análisis del efecto de fuerzas físicas en las complejas geometrías del hueso.

b) La biología molecular que permite detectar la expresión de genes y la síntesis de proteínas tras la aplicación de diferentes fuerzas mecánicas.

c) El avance de la tecnología de imagen, que permite identificar las características micro y nanoestructurales del tejido.

El método informático de elementos finitos (FE, por Finite Element) se trata de un eficaz análisis numérico para estudiar cuantitativamente el comportamiento de una estructura. Este método ha sustituido a los iniciales procedimientos de fotoelasticidad, permitiendo no solo estudiar el predominio de la dirección del estrés sino también cuantificar la intensidad mecánica (3). El objetivo de los FE es asimilar un supuesto comportamiento mecánico numérico al comportamiento biológico de las células de una estructura y de esta manera comparar los resultados de ambas evidencias de manera bidireccional.

A pesar del incremento en el conocimiento de los procesos celulares y moleculares que acontecen en la interfase hueso-implante, poco se conoce sobre el proceso por el cual las fuerzas mecánicas modulan el crecimiento óseo en esa interfase. La mecanotransducción se encarga del estudio de la transmisión y distribución de las señales mecánicas, y su conversión en señales biológicas y químicas dentro de la célula (4).

La respuesta del hueso ante los estímulos mecánicos locales ya fue descrito por Wolff en 1892 (5) estableciendo la hipótesis de que "cada cambio de la forma y la función del hueso, o solo de la función, conlleva cambios definitivos en la arquitectura interna y la conformación externa, siguiendo leyes matemáticas". Posteriormente se ha cuestionado la relación con posibles leyes matemáticas, pero lo que es incuestionable es que las cargas mecánicas no solo afectan el volumen del hueso sino también su organización interna. Desde los trabajos iniciales de Frost $(6,7)$ se ha aceptado de manera general que el hueso se renueva por si mismo a lo largo de la vida mediante las unidades multicelulares básicas (BMUs, por Bone Multicellular (Units), que se forman de grupos de osteoblastos y osteoclastos que primero reabsorben el hueso y posteriormente lo van rellenando por un nuevo tejido. El nuevo hueso formado se organiza en las osteonas del hueso compacto o en las hemi-osteonas o trabéculas del hueso trabecular alineándose según la dirección de las cargas dominantes (8) lo que sugiere que los gradientes de tensión local son un factor regulador de la forma en que se desarrolla el recambio óseo (9) (Fig 1).

Basándose en la observación macroscópica, Wolff (5) estableció el primer planteamiento numérico de la estructura del hueso, con la descripción de la disposición perpendicular de las trabéculas en la cabeza del fémur. Sin embargo, tanto el modelo de Wolf

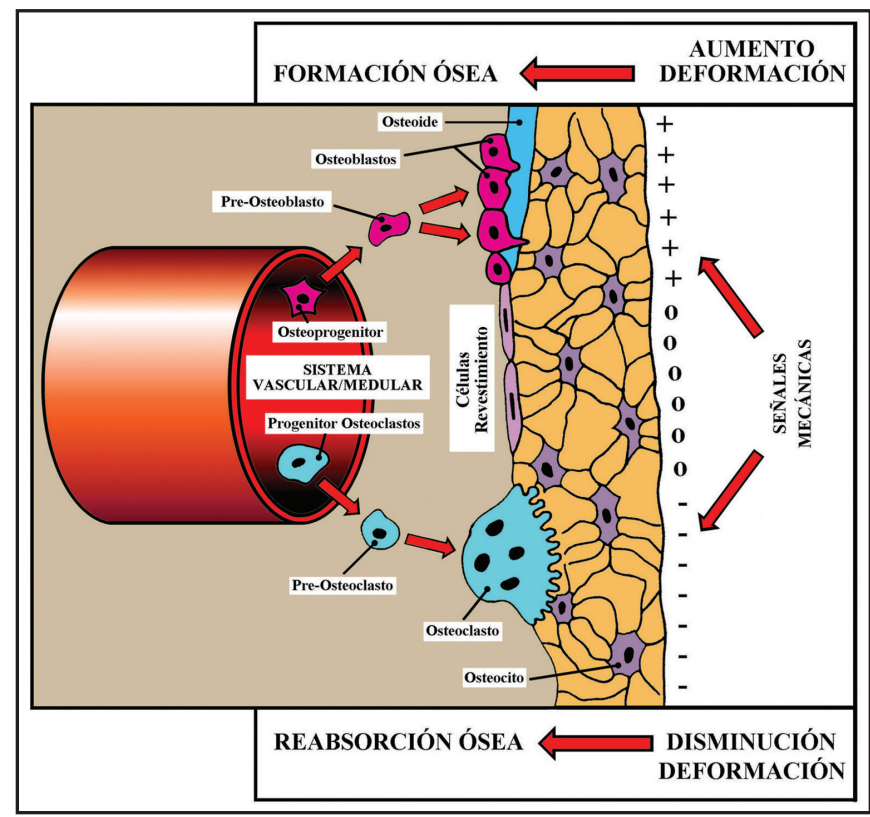

Fig. 1. Esquema del acoplamiento en el remodelado óseo según las cargas aplicadas. 
como el resto de los modelos numéricos posteriores no pueden ser tomados de manera categórica debido al número de factores no mecánicos que también influyen sobre el hueso (genéticos, nutricionales, hormonales, paracrinos, etc). De esta manera se han establecido otros modelos en donde la disposición no es únicamente perpendicular, sino también con otras disposiciones (3). Los modelos finitos tradicionales se han basado en niveles evidentes (macroscópicos) y en la premisa simplista del llamado "supuesto continuo" por el cual se presume un comportamiento similar en cada trabécula ósea. El avance tecnológico de la microtomografia computarizada (micro-CT) y la creación de nuevos algoritmos ha permitido observar el comportamiento individual de una trabécula. Parece que el estudio de elementos microestructurales y la formación posterior de elementos finitos entre 100 millones y 1 billón de elementos finitos establecerán unos modelos mas cercanos a la estructura real (3).

En este artículo se revisan los conceptos generales que se estudian en la mecanobiología y biomecánica de los hueso maxilares. Se establecen las principales propiedades biomecánicas del hueso en las diferentes escalas de medición y determinados factores que influyen en la reacción del hueso perimplantario ante las cargas biomecánicas.

\section{PROPIEDADES BIOMECÁNICAS DEL HUESO (NIVEL MACRO, MICRO Y NANOMÉTRICO)}

Hay que tener en cuenta que durante varios años se han establecido las propiedades biomecánicas del hueso basándose únicamente en la escala macrométrica (escala de centímetros o milímetros), valorando las diferencias estructurales visibles a simple vista que existen entre el hueso trabecular y el hueso cortical (Fig 2). Sin embargo, hoy en día se puede estudiar la estructura del hueso a nivel orgánico, tisular, celular o molecular (10). Las aproximaciones micro y nanoestructurales al hueso están reflejando las diferentes propiedades biomecánicas de los distintos tipos de tejido óseo que se pueden incluir tanto en el hueso trabecular como en el cortical, es decir el hueso reticular, el hueso de fibras paralelas y el hueso laminar. De manera general, en el hueso, la resistencia (resistencia a la deformación) y la rigidez

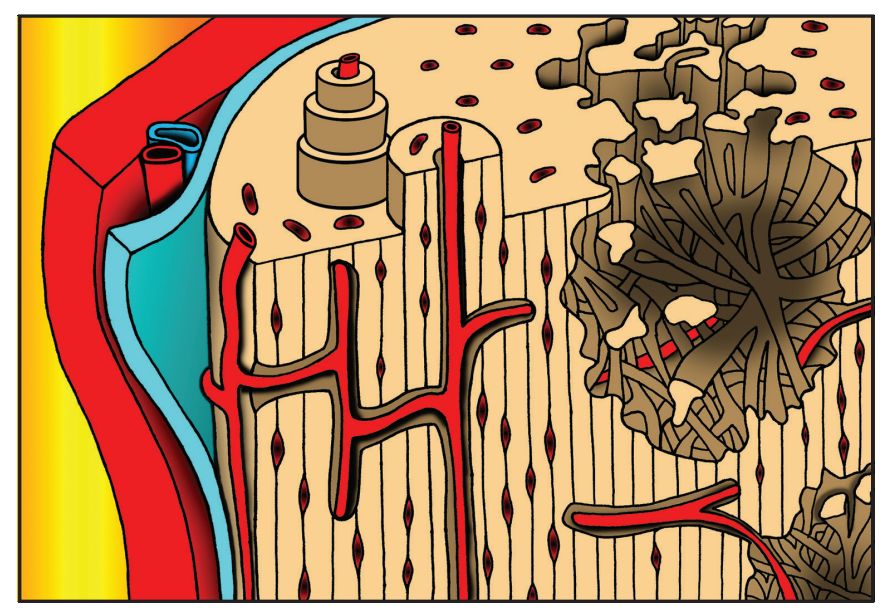

Fig. 2. Estructura trabecular y cortical del hueso.

lo aporta la fase inorgánica, mientras que la tenacidad (resistencia a la fractura) lo aporta la fase orgánica (11).

\section{Nivel macro y micrométrico (órgano/tejido/célula)}

Teniendo en cuenta el carácter general anisotrópico no homogéneo del hueso, el hueso cortical se suele evaluar estructuralmente de manera imprecisa como un material isotrópico (con la misma propiedad biomecánica al ser medida en cualquier dirección), mientras que el hueso trabecular presenta mayor grado de anisotropía en función de las cargas que haya recibido en cada zona (1). Si las propiedades son diferentes en las tres direcciones del espacio se habla de material ortotrópico, mientras que sin las propiedades son iguales en dos direcciones, se habla de isotropía transversal (12).

Las propiedades biomecánicas son variables entre el hueso cortical y trabecular principalmente porque el espacio poroso en el primero es del 5-10\% mientras que en el segundo se encuentra entre $30-90 \%$ (10). La morfología general del hueso, le permite tener una estructura rígida y ligera a la vez, ya que el hueso cortical facilita la rigidez mientras que las trabéculas internas minimizan el peso (1).

Las propiedades mecánicas se han valorado con diferentes métodos como son los ensayos mecánicos, la relación de microdureza Vickers con el módulo de 
elasticidad o las técnicas basadas en ultrasonidos. Actualmente la herramienta más utilizada es la de creación de elementos numéricos o finitos mediante ordenador, que incluso puede añadir elementos reales a partir de imágenes de alta resolución. De manera general, las propiedades biomecánicas del hueso van a depender de su contenido acuoso, de la porosidad (densidad) y del contenido mineral (1).

\section{Hueso Cortical}

Los valores obtenidos para el hueso cortical son menos dispersos que los encontrados en el hueso trabecular. En general, el módulo de elasticidad se encuentra entre 15-20 GPa (13) la resistencia a la tensión se encuentra entre los 80-150 Mpa, y la resistencia a la compresión entre los 90-280 Mpa, debido principalmente al carácter anisotrópico del hueso (1).

Se ha observado que la cortical mandibular es anisotrópica, de tal forma que al igual que la diáfisis femoral, es mas rígida en la dirección longitudinal que en la radial o tangencial, teniendo en estas direcciones un $40-80 \%$ del valor de rigidez que tiene en la dirección longitudinal. La resistencia a la compresión es de 200, 110 y 100 Mpa en la dirección longitudinal, tangencial y radial respectivamente (14).

Por otro lado hay que considerar que los valores de resistencia del hueso cortical son variables en función de la edad. De esta manera la resistencia a la tensión máxima desciende desde los 120 Mpa con 30 años a 70 Mpa a los 100, mientras que el límite máximo de deformación cae del 3,3\% a los 30 años al $1 \%$ a los 100 años. De la misma manera se observan similares efectos en el hueso trabecular (15). Se ha observado en hueso cortical femoral que a partir de los 35 años hay una disminución por década del módulo elástico de $0,35 \mathrm{GPa}$, de resistencia a la flexión de 6,25 Mpa, y de energía de fractura (tenacidad) de $0,03 \mathrm{Kj} / \mathrm{m}^{2}$. El tamaño de las microgrietas también se ha observado aumentado con la edad con valores de $500 \mu \mathrm{m}$ a los 35 años y de hasta 1 $\mathrm{mm}$ de longitud a los 92 años. El aumento del número y tamaño de estas grietas se ha correlacionado positivamente con los resultados de tenacidad del hueso cortical (16).

\section{Hueso Trabecular}

Existen opiniones dispares en cuanto a establecer las propiedades biomecánicas del hueso trabecular. Hay autores que lo consideran con un modulo de elasticidad similar al del hueso cortical (15-20 GPa) (13). Otros autores, sin embargo le adjudican un valor de rigidez menor (0,75-10 GPa) (17). En cualquier caso se han determinado diferentes valores de $E$ para el hueso trabecular en función de la prueba de esfuerzo utilizada y las condiciones del hueso esponjoso (seco, fresco o congelado), aunque siempre con valores menores que el hueso cortical. Los valores evidenciados son muy dispares incluso cuando los datos los emite un mismo autor aplicando el mismo ensayo a diferentes muestras $(1,12)$.

McNamara y cols (18) desarrollaron un modelo sólido obteniendo 4 trabéculas de hueso vivo que posteriormente fueron procesadas con micro-CT y analizadas mediante elementos finitos. Este modelo permite observar la reacción a cargas sobre un modelo con morfología real (incluyendo lagunas de reabsorción activas). El estrés se observaba elevado en el fondo de las lagunas de reabsorción y aumentaría la actividad osteoclástica más allá de lo que inicialmente estaba previsto para renovar el hueso antiguo o dañado, y también evitan la distribución homogénea de la carga a lo largo de la trabécula.

Smit y Burger (19) establecieron la hipótesis que si la deformación baja (desuso) activa los osteoclastos, mientras que si la deformación aumenta (sobrecarga media) se activan los osteoblastos para rellenar la BMU. Estos datos se observaron con unas medidas de estrés no mayores de 16 Mpa y de deformación no mayores de $3.074 \mu \varepsilon$ y con modelos informáticos de trabécula ideales (sin obtenerlas de trabéculas in vivo). Los datos de McNamara y cols (18) establecerían que aunque esto ocurriría habitualmente por debajo de $4.000 \mu \varepsilon$, existirían otros estímulos diferentes para activar a osteoclastos u osteoblastos cuando el estrés y las deformaciones son mayores de $4.000 \mu \varepsilon$.

\section{Nivel nanométrico (molécula)}

Durante muchos años se ha considerado que el hueso cortical y trabecular se consideraba un único 
material desde el punto de vista estructural molecular, y sus diferencias biomecánicas aparecerían en otras escalas debido a la diferencia de densidad. Está hipótesis ha sido rechazado por la evidencia en diferentes estudios de variaciones biomecánicas intrínsecas en los dos tipos de hueso. Si bien los componentes moleculares son idénticos, parece que la disposición de los mismos varía entre ambos influyendo en sus propiedades biomecánicas. Se han observado valores de módulo de elasticidad de 10,4 Gpa en el ensayo de tracción para la trabécula ósea, mientras que en las muestras de hueso cortical se encuentra en 18,6 Gpa (21).

Los dos componentes del hueso tienen propiedades mecánicas extremadamente diferentes. El mineral es duro y frágil, mientras que la proteína (húmeda) es mucho mas blanda pero más resistente (a la tracción, compresión, torsión, etc...). Sin embargo la unión de ambos (composite) combina las propiedades óptimas de cada uno, aportando dureza y resistencia (21). Un factor que va a condicionar las propiedades del hueso cortical o trabecular va a ser la proporción de placas minerales que contenga la matriz colagénica. Se ha observado que después de un periodo de 10-15 días, la matriz colagénica inicia un proceso de mineralización primaria muy rápido (entre varios días y meses dependiendo de la edad y fisiología del individuo) hasta alcanzar un $70 \%$ de su mineralización final (hueso inmaduro entrelazado/ hueso inmaduro de fibras paralelas). El segundo proceso de mineralización que incrementa el resto del $30 \%$ de la mineralización puede llevar varios años y es producto del remodelado óseo (hueso maduro o laminar) $(22,23)$.

Como consecuencia de cada ciclo de remodelado se forman "paquetes de hueso" o BMUs con un contenido mineral diferente según su deposición cronológica (24). Se ha observado también que dentro de una misma trabécula o una misma osteona se presentan diversos contenidos minerales en función de sus diferentes estados biológicos, lo que incrementa la complejidad de su estudio biomecánico (21).

La estructura compuesta del hueso proporciona ciertas ventajas ante las cargas. Las partículas se colocarían con su eje longitudinal de manera paralela a las fibras de colágeno (25). Debido a esas variaciones en las proporciones de colágeno y mineral del hueso, lo más adecuado es considerar las propiedades biomecánicas de ambas estructuras nanométricas por separado. El mineral tiene un modulo elástico de 135 GPa y una deformación de fractura del $0,1 \%$. Los valores para el colágeno son de $1 \mathrm{GPa}$ y $10 \%$ respectivamente. La combinación hace que el hueso tenga unos valores de 10-25 GPa de modulo y 1-1,5\% de deformación (21).

En cuanto al contenido mineral se ha sugerido que el tamaño del cristal, su orientación y su maduración tienen influencia en el comportamiento mecánico final del hueso. La sustitución de iones carbonato por iones hidroxilo o fosfato en las placas minerales, parecen también alterar su estructura cristalina y disminuir la resistencia mecánica (26).

La malla de colágeno distribuida entre las partículas minerales disipa la energía mediante la desviación de las grietas o creando puentes temporales en las grietas creadas y así evitar el fracaso completo. Se ha especulado que el estrés de tensión se transmitiría a través de la malla orgánica (21). Para revelar las propiedades mecánicas del hueso es necesario conocer la distribución de la malla de colágeno. Mediante radiación sincrotrón que permite una resolución intralaminar, se ha observado que el colágeno se dispone en capas de $1 \mu \mathrm{m}$ de espesor con una estructura laminada helicoidal (no ortogonal) sobre si misma cambiando su ángulo de orientación longitudinal de manera regular en $5^{\circ}-25^{\circ}$ en cada capa de fibras. Parece que las estructuras laminadas helicoidales tienen mayor extensibilidad ante compresión o tracción que los laminados ortogonales y se desconoce si esta estructura puede ser modificada ante diferentes tipos de cargas (25).

Con el incremento de edad se ha observado un deterioro de la malla de colágeno incluyendo la pérdida de la capacidad de contracción de las fibras con lo que se disminuyen las propiedades de resistencia. También se observa un aumento en el número de microgrietas y el tamaño de las mismas. Se ha correlacionado las alteraciones en la contracción del colágeno con los resultados de tenacidad, pero no con los de resistencia o rigidez (16). 
Se ha evidenciado también que las microgrietas avanzan con una baja energía de fractura (J-integral) cuando siguen un ángulo paralelo a las fibras de colágeno, mientras que cuando encuentran puentes entre las fibras de colágeno, se desvían en ángulos relativamente agudos en forma de zig-zag, relacionándose con altos niveles de energía y por tanto de tenacidad. La desviación del ángulo de las fibras, sumando a las fibras de colágeno transversales, a las líneas de cementación entre osteonas y al espacio entre laminillas, favorecería la tenacidad del hueso al permitir una mayor absorción de energía (27).

Parece que la existencia de agua dentro del hueso cortical va a influir en la rigidez y tenacidad del mismo. El agua se une a los grupos hidrofílicos del colágeno (glicina, hidroxiprolina, carboxilo e hidroxilisina) y los iones fosfato y calcio de la fase inorgánica. La influencia del agua no solo se refiere al sistema vascular-canalicular sino que parece intervenir en el interior de la matriz ósea. La deshidratación del hueso conlleva una disminución de la tenacidad y la resistencia, lo que podría estar relacionado con las peores propiedades biomecánicas que implica el incremento de la edad (11).

\section{MECANOBIOLOGÍA DEL HUESO PERIMPLANTARIO}

En los últimos años se han impuesto los protocolos de carga que permitan la realización de la rehabilitación prostodóncica lo antes posible. En esta apartado se describen una serie de fundamentos que influyen en la mecanobiología del hueso que rodea a los implantes dentales y que determina el éxito o fracaso de una rehabilitación implantosoportada o implantorretenida.

\section{Carga inmediata, precoz y convencional}

Debido a la controversia existente en relación a los términos aplicado a los protocolos de carga sobre implantes, en el 2003 se emitió una declaración de consenso entre expertos (28) que establecía los siguientes rangos:

a) Carga inmediata es la que se aplica dentro de las 48 horas posteriores a la colocación del implante. b) Carga precoz es la que se aplica entre las 48 horas y los 3 meses después de la colocación del implante.

c) Carga convencional es la que se aplica entre los 3-6 meses después de colocar los implantes.

d) Carga retrasada es la que se aplica mas allá de la carga convencional.

Normalmente la carga retrasada se aplica en los casos que ha existido un procedimiento de regeneración que obliga a alargar el periodo de consolidación. En este consenso se estableció de manera general la posibilidad de realizar carga precoz a las 6 semanas de curación perimplantaria en cualquier situación clínica que presente un hueso tipo 1, 2 ó 3.

Por otro lado, hay que distinguir tres conceptos en cuanto al tipo de carga aplicada:

a) Oclusión directa es la que se consigue cuando la restauración sobre el implante tiene contacto con la dentición antagonista.

b) Oclusión indirecta es la que se consigue cuando la restauración está en anoclusión.

c) Oclusión progresiva es la que se establece de manera "ligera" al inicio de la carga y se va convirtiendo gradualmente en un contacto directo (28).

\section{Patrón masticatorio en pacientes con implantes}

La fuerza de mordida, la eficacia masticatoria y el fluido salivar van a influir en el proceso masticatorio. Hay que tener en cuenta que los dientes realizan un contacto fisiológico durante la masticación y la deglución. Los contactos en máxima intercuspación a lo largo del día no llegan a 18 minutos del total (29). La magnitud de la fuerza que se puede ejercer a nivel oclusal es muy variable según los individuos, aunque lo que si parece establecido es que la fuerza en los sectores posteriores es de 3 a 5 veces mayor que en el sector anterior (30). Existe gran variación entre individuos en cuanto a la frecuencia masticatoria, es decir el número de ciclos masticatorios realizados antes de la deglución del bolo alimenticio. También existen diferencias individuales evidentes en cuanto a la eficacia masticatoria, es decir el grado de fragmentación que se consigue del alimento después de un determinado número de ciclos de masticación (31). 
La masticación es regulada por el sistema nervioso central coordinando la actividad de la mandíbula, la lengua y los músculos faciales. La adaptación de este sistema motor va a ser moldeado a lo largo de la vida del individuo en función de la edad y de la pérdida o modificación dentaria. Parece ser que el único factor estable en un individuo durante el proceso de la masticación es el de la frecuencia masticatoria (número de ciclos masticatorios por unidad de tiempo). La frecuencia masticatoria permanece invariable aunque se modifique la edad o a la dureza del alimento, pero si es influenciada (disminuyéndola) por la pérdida dentaria, con lo que se ha sugerido esa frecuencia como un factor para valorar la eficacia masticatoria (32).

La fuerza de mordida va a depender de varios factores fisiológicos como son la fuerza muscular, la anatomía craneomandibular y el mecanismo de retroalimentación neuromuscular. En pacientes con implantes se han registrado mediciones medias verticales de $200 \mathrm{~N}$ y horizontales de $40 \mathrm{~N}$ (33). La máxima fuerza de mordida vertical que se puede alcanzar con supraestructuras implantosoportadas está alrededor los 800 N (34). Los implantes, a diferencia de los dientes naturales, se encuentran anquilosados en el hueso sin un ligamento periodontal que aporta mecanorreceptores y una función de absorción de cargas. De esta manera, se ha fomentado en la literatura durante décadas la importancia del control biomecánico de los implantes por medio de una oclusión adecuada y evitando las cargas laterales.

Entre varias diferencias, los dientes naturales presentan un fulcro de palanca en el tercio apical cuando son sometidos a una carga lateral, mientras que los implantes lo tienen en la cresta ósea, lo que explica la concentración de estrés en esa zona y ciertas reabsorciones en esa localización (35). Otra diferencia entre los dientes naturales y los implantes es su capacidad de movimiento en su alveolo/lecho óseo. De tal manera que los dientes permiten una intrusión axial de 25-100 $\mu \mathrm{m}$, mientras que en los implantes solo se permiten 3-5 $\mu \mathrm{m}$ (35). Cuando la carga es aplicada lateralmente los dientes permiten una movilidad de 56-108 $\mu \mathrm{m}$ antes de iniciar una rotación sobre su ápice (36), mientras que los implantes alcanza un movilidad lateral de 10-50 $\mu \mathrm{m}$ sin producirse ninguna rotación (35). Por otro lado el movimiento en los dientes sigue 2 fases, la primera dependiente del ligamento que es compleja y no-lineal y una segunda lineal y dependiente del hueso, mientras que en los implantes es únicamente en una fase, lineal y dependiente de la elasticidad del hueso (35).

Existen numerosos factores que afectan la carga que reciben los implantes (número de implantes, macrodiseño, superficie, tipo de prótesis, ajuste de estructura, etc) (37). Esta gran variabilidad de factores, sumado a las diferencias en cuanto a el patrón masticatorio (fuerza de mordida, eficacia masticatoria y fluido salival) y los hábitos alimenticios, hacen realmente imposible establecer a la carga de implantes inmediata/precoz como una carga controlada y cuantificable.

\section{Concepto de carga estática, cuasi-estática, dinámica y cíclica}

Se considera carga estática a la que se aplica sobre una estructura de forma continua durante un tiempo determinado (p.ej. los ensayos de tracción, compresión, etc...). El concepto de carga cuasi-estática (quasistatic) hace referencia a la aplicación de una carga de manera continua durante un tiempo determinado, pero distribuida en ciclos de determinada frecuencia. La carga dinámica hace referencia a una aplicación súbita de la carga, donde el tiempo no tiene prácticamente valor (p. ej. los ensayos por impacto y de fatiga). Las cargas cíclicas se caracterizan por la repetición reiterativa de un estímulo de carga (estática o dinámica) por unidad de tiempo (ciclos) $(38,39)$. En este sentido puede haber cargas cíclicas-estáticas (cuasi-estáticas) y cargas cíclicasdinámicas.

Los movimientos de la masticación, considerados de manera unitaria se considerarían como carga dinámica y en su conjunto como una carga cíclicadinámica. La sobrecarga oclusal se asimila a una carga dinámica descontrolada, que en varios aspectos se puede considerar como cíclica, ya que se aplica a intervalos pero sin embargo no a una frecuencia determinada (38). En relación al complejo huesoimplante-prótesis, se ha definido la carga estática 
como la resultante de las fuerzas aplicadas sobre dicho complejo antes de aplicar cualquier tipo de carga oclusal. La carga dinámica hace referencia a la resultante de fuerzas a las que se somete al complejo durante la función masticatoria.

Parece que las tensiones generadas como consecuencia de un ajuste pasivo inadecuado (carga estática) son más nocivas sobre las juntas protésicas que sobre la unión hueso implante. De esta manera se ha observado que un importante desajuste del sistema implante-pilares crea desplazamientos y aflojamientos de los componentes de la prótesis e incluso fractura del propio implante pero escasos efectos sobre el hueso perimplantario (40). A pesar de la controversia que existe sobre la sobrecarga en los implantes y el diseño de cantilevers, existe la tendencia general a pensar que la fuerza oclusal patológica y la distribución desfavorable de los puntos de contacto pueden llevar a una alta susceptibilidad a la pérdida ósea, fractura del implante y fallos protésicos (41). De hecho la pérdida de osteointegración y la pérdida de hueso marginal debido a sobrecarga oclusal fueron descritas experimentalmente en los trabajos de Isidor y cols. con primates no humanos, aunque las condiciones de este experimento no eran las mas realistas posibles debido a las elevadas prematuridades aplicadas $(42,43)$. Por otro lado, hay autores que aluden a la falta de evidencia de la sobrecarga como causa de la reabsorción ósea y consideran que la principal causa de la pérdida ósea marginal perimplantaria no es la sobrecarga sino la infección perimplantaria (44).

La frecuencia de aplicación de la carga se ha visto determinante en la respuesta anabólica tanto en los linajes del esqueleto apendicular como el esqueleto craneofacial (Fig. 3). De tal manera que la carga dinámica aplicada mediante oscilación cíclica presenta mejores resultados anabólicos que la carga estática aplicada a la misma magnitud y duración $(45,46)$. Estudios experimentales han demostrado que el índice de carga (la frecuencia) es más importante que la amplitud de carga (la cantidad) para inducir la formación de hueso. De hecho, se ha visto que un carga de baja amplitud $(<10 \mu \varepsilon)$ a alta frecuencia (10-100 Hz) es capaz de estimular crecimiento óseo e inhibir la osteoporosis por desuso (47).

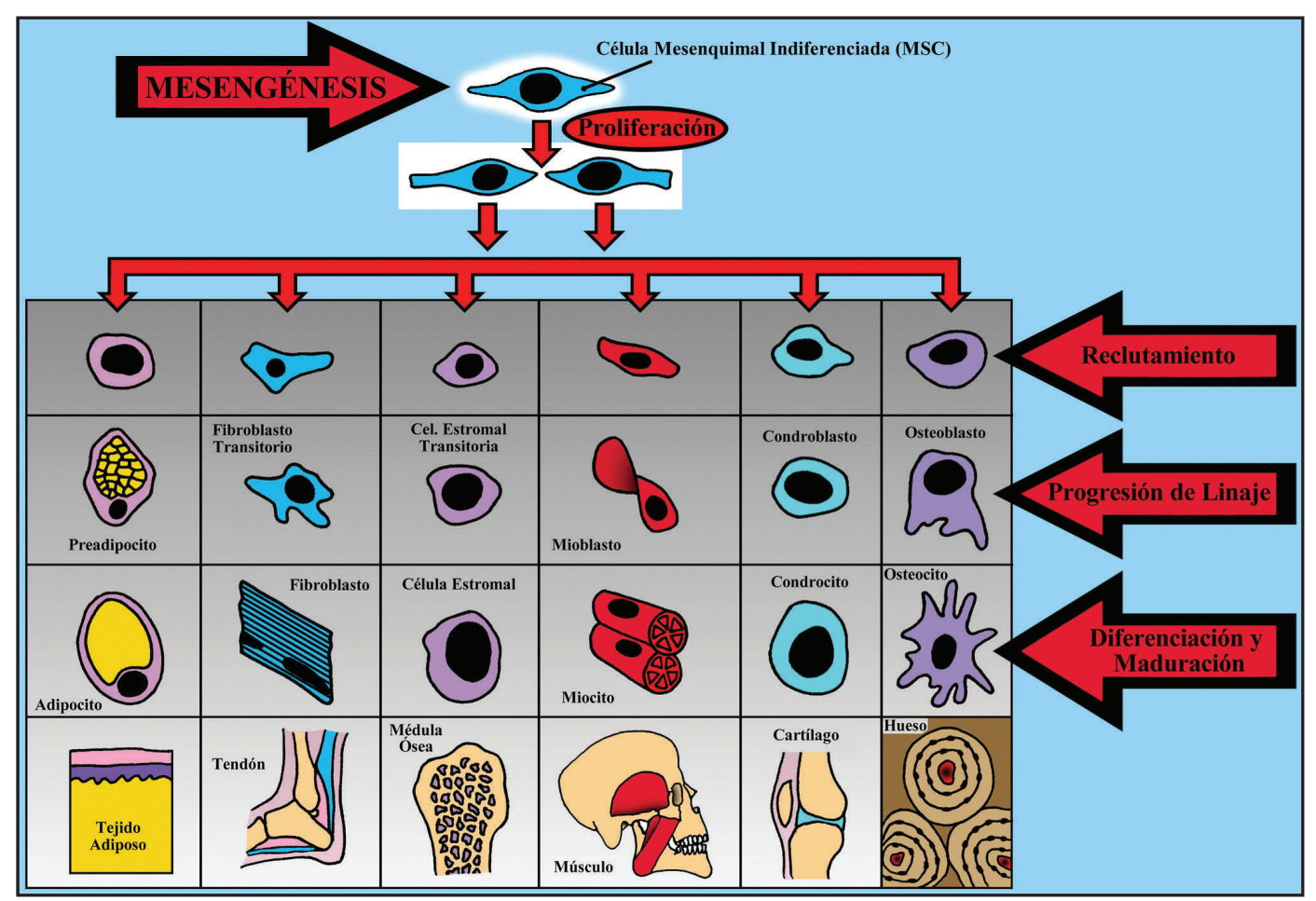

Fig. 3. Derivación del linaje a partir de células mesenquimales. 


\section{Biomecánica implantológica}

En la aplicación de carga ortodóncica, tanto con anclaje de implantes como anclaje dentario, se aplica la tercera ley de Newton, la cual establece que "la interacción de dos cuerpos es siempre igual y en dirección opuesta". Es decir cuando se utiliza una estructura como anclaje para ejercer una carga hay que tener en cuenta las fuerzas de reacción que actuarán. La fuerza que se ejerce se representa por un vector de cantidad que tiene tanto magnitud como dirección (48).

La fuerza ejercida sobre la cabeza de un implante va a producir un momento el cual es un vector de cantidad que tiende a producir rotación del cuerpo sobre un eje. Un caso especial de momento es la idea de momento-par que se forma por un par de fuerzas no colineales de sentido opuesto actuando sobre un cuerpo, que originan una resultante de fuerza cero aunque si se produce un efecto sobre el cuerpo (48).

Cuando se aplica una fuerza lateral sobre un diente o un implante, la fuerza $(F)$ aplicada sobre la cabeza del implante tiende a rotar el mismo sobre un centro de rotación (CR). La tendencia a la inclinación se expresa por el momento, y su magnitud será:

$$
M=F \cdot d
$$

donde $d$ es la distancia entre la línea de acción de $F$ y el punto CR. Las unidades habituales de medida del momento son $\mathrm{Nm}$ o $\mathrm{Ncm}$.

Cuando se aplica una fuerza lateral sobre la cabeza de un implante en clínica, se produce un vector momento tridimensional, que tenderá a producir inclinación o rotación sobre tres ejes perpendiculares (mesiodistal, bucolingual y oclusoapical). Si se quisiera mover lateralmente todo el implante (no solo inclinarlo) habría que ejercer una fuerza lateral sobre su CR lo que es físicamente imposible. La solución consistiría en aplicar una fuerza sobre la cabeza del implante y aplicar un momento par que contrarreste, consiguiendo un momento neto de cero y un movimiento de translación. Sin embargo, parece que el ligamento periodontal juega un papel fundamental como interfase continua de adaptación, que permite la translación de los dientes, pero no ocurre lo mismo en los implantes o los dientes anquilosados. De hecho solo un $10 \%$ de integración es suficiente para evitar esa translación de un implante o un diente anquilosado. En los implantes, las cargas ortodoncias producen un aumento intenso del remodelado pero sin generar un movimiento (49).

La carga que se aplica habitualmente cuando se usa un implante como anclaje ortodóncico no suele sobrepasar algunos newtons (alrededor de $5 \mathrm{~N}$ y momentos de $2 \mathrm{Ncm}$ ), mientras que las cargas masticatorias son mucho más elevadas, alcanzando momentos del rango de 10 a $40 \mathrm{Ncm}$. De hecho una vez conseguida la osteointegración se han registrado fracasos por fatiga en el rango de 1.000-1.500 N en dirección axial y de 200-300 N en dirección lateral, lo que indica niveles muy por encima de los utilizados en el anclaje ortodóncico (48).

\section{BIBLIOGRAFÍA}

1. Rincón E, Ros A, Claramunt R, Arranz F. Caracterización mecánica del material óseo. Rev Tec Des 2004; 2: 0-27.

2. Van der Meulen MCB, Huiskes R. Why mechanobiology?. A survey article. J Biomech 2002; 35: 401-4.

3. Jacobs CR. The mechanobiology of cancellous bone structural adaptation. J Rehab Res Dev 2000; 36(2):209-16.

4. Lim CT, Zhou EH, Quek ST. Mechanical models for living cells. A review. J Biomech 2006; 39:195216.

5. Wolff J. eds. The law of bone remodelling. Berlin: Springer (translation of the German 1892 edition) 1986.

6. Frost HM, eds. Mathematical elements of bone remodelling. Springfield: Charles C. Thomas Publisher, 1964.

7. Frost HM. Bone mass and the mechanostat: a proposal. Anatomical Record 1987; 219: 1-9. 
8. Parfitt AM. Osteonal and hemi-osteonal remodeling: the spatial and temporal framework for signal traffic in adult human bone. Journal of Cellular Biochemistry 1994; 55: 273286.

9. Cano J, Campo J, Gonzalo JC, Bascones A. Consolidation period in alveolar distraction: A pilot histomorphometric study in the mandible of beagle dogs. Int J Oral Maxillofac Impl 2006; 21(3):380-91.

10. Carter DR, Beaupre GS. Skeletal tissue histomorphology and mechanics. En Carter DR, Beaupre GS. Skeletal function and form. Mechanobiology of skeletal development, aging and regeneration. Cambridge: Cambridge University Press 2001: 31-48.

11. Nyman JS, Anuradha R, Shen X, Acuna RL, Tyler $\mathrm{JH}$, Wang $\mathrm{X}$. The influence of water removal on the strength and toughness of cortical bone. J Biomech 2006; 39: 931-8.

12. Van Eijden TM. Biomechanics of the mandible. Crit Rev Oral Biol Med 2000; 11(19): 123-36.

13. Rho JY, Tsui TY, Pharr GM. Elastic properties of human cortical and trabecular lamellar bone measured by nanoindentation. Biomaterials 1997; 18:1325-30.

14. Dechow PC, Nail GA, Schwartz-Dabney CL, Asman RB. Elastic properties of human supraorbital and mandibular bone. Am J Phys Anthropol 1993; 90:291-306.

15. McCalden RW, McGeough JA, Barker MB, CourtBrown CM. Age-related changes in the tensile properties of cortical bone. J Bone Joint Surgery 1993; 75-A: 1193-205.

16. Zioupos P. Ageing human bone: Factors affecting its biomechanical properties and the role of collagen. J Biomat Appl 2001b; 15: 187-229.

17. Nicholson PH, Cheng XG, Lowet G, Boonen S, Davie MW, Dequeker J, Van der Perre G. Structural and material mechganical properties of human vertebral cancellous bone. Med Eng Phys 1997; 19: 729-37.

18. McNamara LM, Van der Linden JC, Weinans $H$, Prendergast PJ. Stress-concentring effect of resorption lacunae in trabecular bone. J Biomech 2006: 39: 734-741.

19. Smit TH. Burger EH,. Is BMU-coupling a strainregulated phenomenon? A finite element analysis. J Bone Min Res 2000;15: 301-7.

20. Rho JY, Ashman RB, Turner ChH. "Young's mudulus of trabecular and cortical bone material: ultrasonic and microtensile measurements". Journal of Biomechanics 1993; 26 (2): 111-9.

21. Frantzl P, Gupta HS, Paschalis EP, Roschger P. Structure and mechanical quality of the collagenmineral nano-composite in bone. J Mater Chem 2004; 14: 2115-23.

22. Cano J, Bascones A, Campo J. Biología de la regeneración ósea. En Bascones A, Cano J, Campo J, Chiapasco M, eds. Distracción alveolar histogénica. Aplicada a la implantología oral. Madrid: Avances. 2006: 9-37.

23. Sommerfeldt DW, Rubin CT. Biology of bone and how it orchetrates the form and function of the skeleton. Eur Spine J 2001; 10: S86-S95.

24. Akkus O, Polyakova-Akkus A, AdarF, Schaffler MB. Aging of microstructural compartments in human compact bone. J Bone Min Res 2003;18 (6):1012-9.

25. Wagermaier W, Gupta HS, Gourrier A, Burghammer M, Roschger P, Fratzl P. Spiral twisting of fiber orientation inside bone lamellae. Biointerphases 2006; 1:1-5.

26. Busa B, Miller LM, Rubin CT, Qin YX, Judex S. Rapid establishment of chemical and mechanical properties during lamellar bone formation. Calcif Tissue Int 2005; 77: 386-94.

27. Peterlik H, Roschger P, Klaushoffer K, Fratzl P. Orientation dependent toughness of lamellar bone. Int J Fract 2006; 139:395-405. 
28. Cochran DL, Morton D, Weber HP. Consensus statements and recommended clinical procedures regarding loading protocols for endosseous dental implants. Int J Oral Maxillofac Imp 2004; 19 suppl: $109-13$.

29. Graf H, Zander HA. Tooth contact patterns in mastication. J Prosthet Dent 1963; 13:105566.

30. Hagberg C. Assesments of bite force: a review. J Craniomand Dis 1987;1:162-9.

31. Fontijn-Tekamp FA, Van der Bilt A, Abbink JH, Bosman F. Swallowing threshold and masticatory performance in dentate adults. Physiol Behav 2004;83:431-6.

32. Peyron MA, Blanc O, Lund JP,Woda A. Influence of age on adaptability of human mastication. J Neurophysiol 2004;92:773-9.

33. Morneburg TR, Proschel PA. In vivo forces on implants influenced by occlusal scheme and food consistency. Int J Prosthodont 2003;16:481-6.

34. Van Eijden TM. Three-dimensional analysis of human bite-force magnitude and moments. Arch Oral Biol 1991;36:535-9.

35. Sekine H, Komiyama Y, Hotta H. Yoshida K. Mobility characteristics and tactile sensitivity of osseointegrated fixture-supporting systems. En: Van Steenberghe D, eds. Tissue integration in oral maxillofacial reconstruction; 1986; Amsterdam: Excerpta Medica:326-32.

36. Hillam DG. Stresses in the periodontal ligament. Journal of Periodontal Research 1973;8:51-6.

37. Sahin S, Cehreli MC, Yalcin E. The influence of functional forces on the biomechanics of implnatsupported prostheses. A review. J dent 2002;30: 271-82.

38. Vega JM. Propiedades físicas mecánicas de los materiales odontológicos (I): principios generales sobre ensayos tecnológicos aplicados a los materiales dentales. En Vega JM, eds. Materiales en odontología. Fundamentos biológicos, clínicos, biofísicos y fisicoquímicos. Madrid: Avances. 1996:161-75.

39. Martin DE, Severns AE. Determination of mechanical stiffness of bone by PQCT measurments: correlation with nondestructive mechanical four-point bending teat data. J Biomech 2004;37:1289-93.

40. Jemt T, Lekholm U. Measurements of bone and frame-work deformations induced by misfit of implant superstructures. A plito study in rabbits; Clin Oral Imp Res 1998;9:272-80.

41. Kim Y, Oh Tj, Misch CE, Wang HL. Occlusal considerations in implant therapy: clinical guidelines with biomechanical rationale. Clin Oral Impl Res 2005;16:26-35.

42. Isidor F. Loss of osseointegration caused by occlusal load of oral implants. A clinical and radiographic study in monkeys. Clinical Oral Implants Research 1996; 7:143-52.

43. Isidor F. Histological evaluation of periimplant bone at implants subjected to occlusal overload or plaque accumulation. Clinical Oral Implants Research 1997;8:1-9.

44. Lang NP, Wilson TG, Corbet EF. Biological complications with dental implants: their prevention, diagnosis and treatment. Clinical Oral Implants Research 2000; 11 (Suppl.): 146-55.

45. Collins JM, Ramamoorthy K, Da Silveira A, Patston PA and Mao JJ.Microstrain in intramembranous bones induces altered gene expression of MMP1 and MMP2 in the rat. J Biomechanics 2005;38:485-92.

46. Szmukler-Moncler S, Piatelli A, Favero GA, Dubruille JH. Considerations preliminary to the application of early and immediate loading protocols in dental implantology. Clin Oral Impl Res 2000;11:12-25.

47 Rubin CT, Sommerfeldt DW, Judex S, Qin YX. Inhibition of osteopenia by low magnitude, high- 
frequency mechanical stimuli. Drug Discov Today 2001;6:848-58.

48. Brunski JB, Slack JM. Carga ortodóncica en los implantes: consideraciones biomecánicas. En Higuchi KW, eds. Aplicaciones ortodóncicas en implantes osteintegrados. Caracas: AMOLCA; 2002: 89-109.

49. Roberts E. Anclaje ortodóncico con implantes osteintegrados: fisiología ósea, metabolismo y biomecánica. En Higuchi KW, eds. Aplicaciones ortodóncicas en implantes osteintegrados. Caracas: AMOLCA; 2002: 161-91.

\section{CORRESPONDENCIA}

Jorge Cano

Departamento de Medicina y Cirugía Bucofacial Facultad de Odontología. Universidad Complutense 28040 Madrid

Email: jo.cano@wanadoo.es 